\title{
Management of cytokine release syndrome: an update on emerging antigen-specific T cell engaging immunotherapies
}

\author{
Roman H Khadka1,2, Reona Sakemura³, Saad S Kenderian*,2,3 \& Aaron J Johnson**,2,4 \\ ${ }^{1}$ Mayo Clinic Graduate School of Biomedical Sciences, Mayo Clinic, 200 First St SW, Rochester, MN 55905, USA \\ ${ }^{2}$ Department of Immunology, Mayo Clinic, 200 First St SW, Rochester, MN 55905, USA \\ ${ }^{3}$ Division of Hematology, Mayo Clinic, Rochester, MN 55905, USA \\ ${ }^{4}$ Department of Neurology, Mayo Clinic, 200 First St SW, Rochester, MN 55905, USA \\ *Author for correspondence: Kenderian.Saad@mayo.edu \\ **Author for correspondence: Johnson.Aaron2@mayo.edu
}

First draft submitted: 6 May 2019; Accepted for publication: 22 May 2019; Published online: 5 June 2019

Keywords: bispecific T cell engager $\bullet$ BiTE $\bullet$ CAR T cells $\bullet$ chimeric antigen receptor $\bullet$ CRS $\bullet$ cytokine release syndrome $\bullet$ immunotherapy $\bullet$ neurotoxicity

Antigen-specific immunotherapies have immense potential to harness and fortify the inherent antitumor capacity of the immune system. Due to their unparalleled success in recent years, antigen-targeting immunotherapies such as chimeric antigen receptor (CAR) T cells [1,2] and bi-specific T cell engagers (BiTEs) [2-5] have become state-ofthe-art approaches for treating hematological malignancies. Although a significant portion of the innovation and engineering has been invested toward improving the efficacy and potency of these $T$ cell-engaging immunotherapies, these therapeutic strategies are prone to varying degrees of toxicities, including cytokine release syndrome (CRS) and neurotoxicity syndrome. For ensuring success of these promising and revolutionary therapies, both systematic grading and therapeutic management of CRS are essential for the field of immuno-oncology.

In light of existing variability in assessment and management of toxicities across clinical centers, the American Society for Transplantation and Cellular Therapy (ASTCT) has released a consensus report for grading and management of CRS and neurotoxicity associated with immune effector cell therapies [6]. These consensus recommendations aim to standardize evaluation of therapeutic products employed in clinical trials across institutions. ASTCT has now defined CRS as a supraphysiologic response following any immune therapy that results in the activation or engagement of endogenous or infused $\mathrm{T}$ cells and/or other immune effector cells with symptoms that must include fever at the onset, and may include hypotension, capillary leak and end organ dysfunction [6]. This collaborative effort has been heralded as a step forward in management of toxicities. The purpose of this commentary is not recommendatory of specific strategies, nor does it demand revision on established consensus but rather provide an updated view on breakthroughs in CRS management (Figure 1) based on cytokine-targeting (IL-6, granulocyte-macrophage colony-stimulating factor [GM-CSF], IL-1 $\beta$ ) [7-9] and engineering concepts (inhibitory CAR, CRISPR GM-CSF ${ }^{\mathrm{K} / \mathrm{O}}$ ) $[2,8]$ in both preclinical models and human studies. Herein, we will therefore address recent developments in the field of CRS management and limitations faced by current measures.

\section{Emerging T cell-engaging immunotherapies associated with CRS}

Though 'on-target, off tumor' toxicities and CRS have been previously reported following treatment strategies with CD40 agonists, immune-checkpoint inhibitors and cytotoxic T cell lymphocyte (CTL) therapy, new forms of immunotherapies including CAR T cells and BiTEs present unique form of CRS accompanied by neurotoxicity. This further necessitates better understanding on ways to overcome toxicities associated with each as discussed below: 


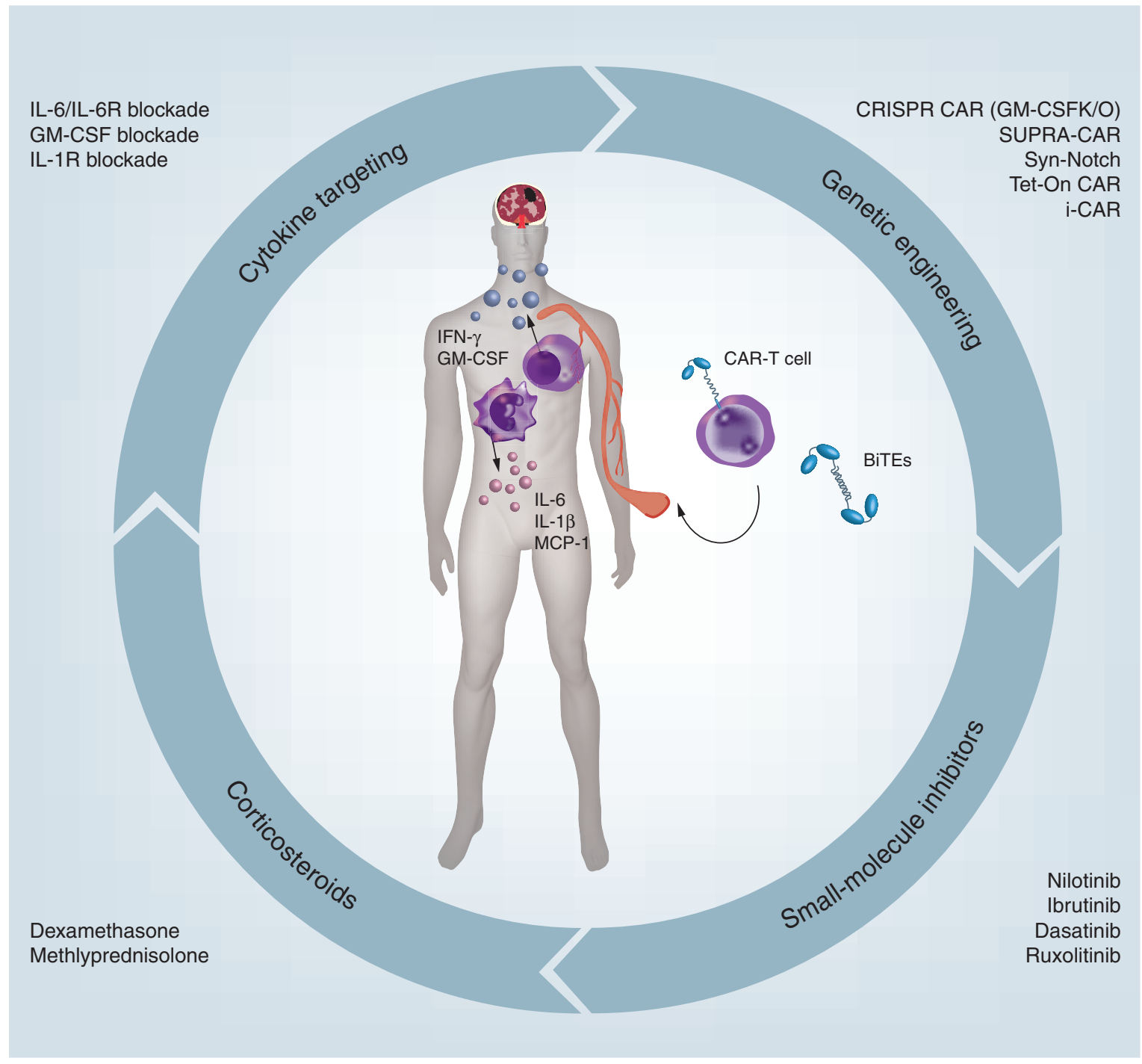

Figure 1. Management of cytokine release syndrome and neurotoxicity associated with T cell engaging immunotherapies. Infusion of immunotherapies such as CAR T cells and BiTEs can lead to acute toxicities that is characterized by elevated levels of pro-inflammatory cytokines in patients. Upon activation post-therapy, $T$ cells can facilitate differentiation and proliferation of myeloid cells by secretion of GM-CSF and IFN- $\gamma$. Licensed monocytes and macrophages can secrete IL- 6 and IL-1 $\beta$ further contributing to systemic toxicity. Subsequently, vascular permeability and disruption of blood-brain barrier follow aberrant local and systemic activation of myeloid cells that can result in neurotoxicity syndrome. Genetic engineering approaches such as CRISPR CAR, SUPRA-CAR, inhibitory CAR (iCAR) and others incorporate built-in safety features to prevent CRS and neurotoxicity. Additionally, cytokine targeting approaches (monoclonal antibody, receptor antagonists), small molecule inhibitors and corticosteroids can be employed as therapeutic interventions to counteract toxicities that occur after infusion of immune cell engaging immunotherapies.

BiTE: Bispecific T cell engager; CAR: Chimeric antigen receptor; GM-CSF: Granulocyte-monocyte colony-stimulating factor; iCAR: Inhibitory CAR; MCP-1: Monocyte chemoattractant protein-1; SUPRA: Split, universal and programmable.

\section{Chimeric-antigen receptor T cells}

Recent US FDA approval of Tisagenlecleucel (KYMIRAH $\left.{ }^{\circledR}\right)$ and Axicabtagene ciloleucel $\left(\right.$ YESCARTA $\left.^{T M}\right)$ highlights the contribution made by CAR T cells for treating B-cell malignancies. Despite being at the forefront of effective cancer treatment, CAR T cells frequently exhibit severe to life-threatening toxicities in clinical trials [10-12]. Aside from B-aplasia and neurotoxicity, CRS is the predominant toxicity associated with current B-cell targeting CAR T cells employed in clinical studies. Knowledge gained from recent clinical trials suggests that CRS is characterized by flu-like symptoms which advance into a shock-like syndrome with massive elevation in cytokines. There is 
variability in kinetics and exact timing of CRS induction. Nevertheless, there is a general consensus that patients exhibit fever, vascular leakage and some degree of organ dysfunction $[6,13]$.

Due to elevation of the signature set of CRS-related cytokines including IL-6, IL-10, TNF- $\alpha$, C-reactive protein (CRP) and IFN- $\gamma$ following CAR T cell therapy, it has invited comparisons with toxicities observed in prior studies involving TGN1412, a CD28-targeting super agonist monoclonal antibody, where the agonist led to rapid onset of life-threatening toxicities [6]. The use of CD28 over 4-1BB as the costimulatory domains in CAR T cells is known to lower activation threshold and thus has been speculated to influence severity of CRS [1]. A recent preclinical study using xenograft model showed no difference in toxicity between CD19-specific CAR T cells with CD285 domain compared with 4-1BBל [7]. Though in the same study, investigators revealed targeting CD44v6 antigen

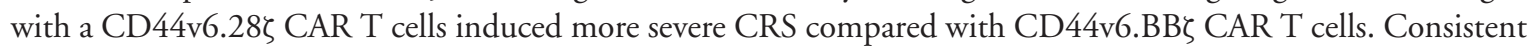
with aforementioned preclinical study, comparable CRS and neurotoxicity have been reported for both 4-1BB and CD28 CAR- constructs in patients $[1,7]$ though direct clinical evidence is limited. Meanwhile, similar association has been predicted between transmembrane/ hinge regions and toxicities. Thus, inadequate comparative studies on the effect of CAR constructs in promoting toxicities warrants further investigation. Besides CAR construct, additional factors such as conditioning regimen [13], disease burden as well as antigen of interest [7] may affect the outcome of CRS toxicities following CAR T cell infusion.

Amidst all the unresolved aspects of CRS, it has however become increasingly apparent that myeloid cells are critical in facilitating progression of CRS as well as neurotoxicity syndrome manifested in both preclinical [7,9] and clinical studies [10-13]. Expression of myeloid cell recruitment chemokines (MIP-1 alpha and MCP-1) concurrently with differentiating cytokines (IFN- $\gamma$, GM-CSF, IL-6) secreted by CAR T cells have been closely associated with severity of CRS in patients $[1,10,11,13]$. In early stages of CRS, GM-CSF can contribute to the differentiation of inflammatory myeloid progenitors. Similarly, GM-CSF is associated with autoimmune diseases such as rheumatoid arthritis (RA) that is characterized by aberrant immune activation and exhibition of features observed in CRS. Correspondingly, two independent murine studies have also highlighted the role of macrophages and monocytesderived IL- 6 and IL-1 $[7,9]$. These targetable cytokines are elevated in the serum of CRS patients experiencing severe CRS following CAR T cell infusion [11-13].

\section{Bi-specific T cell engagers}

BiTEs are another synthetic therapy that incorporate two single chain variable fragments (Sc-Fvs) connected with a flexible short amino acid linker that targets distinct antigens on T cell and tumor cells in tandem. Despite lack of active trafficking abilities possessed by CAR T cells, BiTE has shown promising efficacy against relapsed or refractory B-cell acute lymphoblastic leukemia in multicenter Phase II [4] and Phase III [5] trials. Often overshadowed by current breakthroughs with CAR T cells that employ shared Sc-Fv engineering principles, blinatumomab (CD19directed BiTE) has also successfully acquired US FDA approval for the treatment of relapsed/refractory B-cell acute lymphoblastic leukemia. In theory, blinatumomab activity is independent of in vivo T cell expansion and confers advantage over CAR T cells in a hostile immunosuppressive tumor microenvironment. However, both BiTEs and CAR $\mathrm{T}$ cells have produced dismal efficacy in promoting immunity against solid-tumors. Unfortunately, early clinical studies designed to treat non-Hodgkin lymphoma with blinatumomab resulted in CRS and neurological symptoms including tremors and convulsions in patients. Subsequent trials that followed have also displayed neurological deficits that have mostly been manageable [4]. Solid-tumor antigens targeted using BiTEs include prostate-specific membrane antigen, HER family, EPCAM and gpc3, among these catumaxomab (CD3-coupled EPCAM-specific BiTE) used to treat malignant ascites in a Phase I trial resulted in hepatotoxicity and CRS [14]. Therefore, the therapeutic gains made by BiTEs are hindered by considerable toxicities that will require improved management strategies in the clinic.

\section{Neurotoxicity: temporal \& pathophysiological relationship with CRS}

Neurotoxicity is a life-threatening complication associated with CAR T cells and BiTEs. Pertaining to CRS, the ASTCT has defined a consensus grading system for neurotoxicity, which has been termed immune effector cellassociated neurotoxicity syndrome (ICANS) to account for its unique pathophysiology such as cerebral edema and standardization of the care for future immunotherapies. Manifestation of neurotoxicity syndrome has varied across studies due to variability in models, manufacturing and grading measures [1,6]. Nevertheless, multiple studies do concur that strong antitumor response is displayed in most cases of neurotoxicity syndrome. 
Often in patients with severe CRS symptoms culminate to delirium, seizures and encephalopathy $[1,13]$. Though the temporal aspect of CRS and onset of neurotoxicity syndrome is still enigmatic, ASTCT has determined that grade of CRS occurring concurrently, or preceding neurotoxicity is less informative to compare ongoing trials across the nation [6]. Rather participating investigators in 2018, ASTCT meeting jointly concluded that defining the nature of neurotoxicity independently will offer benefits in managing the syndrome.

Disruption of blood-brain barrier (BBB) is another prominent feature that accompanies neurotoxicity syndrome $[1,8,13]$. Evidence of endothelium activation (alongside dysregulation in Angiopoietin-2) and brainstem hemorrhage has been reported in patients who succumbed to severe case of neurotoxicity [13]. Leakiness of the vasculature is associated with elevated IL-6, IFN- $\gamma$ MCP-1/CCL2 and GM-CSF in the CSF. Increased leukocyte recruitment and neuroinflammation was also described in some patients. Based on these clinical observations, our group has recently modeled BBB disruption using MRI imaging in ALL-patient derived xenograft model to instigate basic research into the mechanism of this syndrome [8].

The significance of understanding the mechanism of ICANS alongside CRS is reinforced by the fact that neurotoxicity syndrome is not just limited to anti-CD19 CAR T cell therapies. Recently, the first-in-human antiBCMA CAR T cell trial for multiple myeloma [15] revealed evidence of encephalopathy and mild neurological deficit in a patient experiencing severe CRS. Yet it must be stressed that the investigators clearly state neurological toxicity was lower compared with that seen in anti-CD19 CAR T cell trials [15]. Due to the temporal and pathological relationship between severe CRS and ICANS, further investigations to define the underlying factors that are shared among these syndromes will continue to be an active area of research.

\section{Management of CRS: current perspective}

\section{IL-6 \& IL-6R antagonism}

IL-6 has emerged as a critical cytokine in exacerbating the progression of CRS in T cell-engaging therapies. The significance of this effector cytokine has been increasingly evident in CAR T cell therapies as recent studies in both experimental models [7,9] and clinical trials [10-12] demonstrate its pathological role. Clinicians and researchers alike have recognized parallels between CRS and macrophage activation syndrome due to shared signature cytokine profile as well as cellular mediators [6]. Consequently, much of the recent research efforts have further verified involvement of myeloid lineage in driving the pathology via IL-6. Furthermore, targeting IL-6 may also have utility in restricting vascular leakage considering that endothelial cells contribute to IL- 6 production in patients and may correlate with coagulopathy, hypotension and ultimately, severity of CRS [13]. While numerous preclinical studies have confirmed myeloid cells including monocytes and macrophages as the primary source of IL-6 in both CAR T cell therapy as well as blinatumomab $[3,16]$, the role of nonimmune mediators has been fairly understudied in this context which calls for additional inquiry in such directions.

Depending on the clinical center both tocilizumab (IL-6 receptor targeting monoclonal antibody) and siltuximab (chimeric anti-IL-6 monoclonal antibody) have been used as approaches to control CRS [1]. In light of approval of tocilizumab for management of CRS by both US FDA and European Medicines Agency, there have been concerns raised about possible elevation of IL-6 due to blockade of the receptor that subsequently lead to accumulation of this effector cytokine in the CNS. Therefore, the prospect of siltuximab being included to intervene CRS is probable based on the rationale that it can lead to favorable outcome in cases of passive diffusion of IL-6 into the CNS [1]. Currently, the greater effectiveness of siltuximab over tocilizumab is highly debatable [1], and these concerns do highlight the need for direct comparative studies that elucidate the efficacy of tocilizumab and siltuximab. Nevertheless, strategies to target IL-6 have been particularly effective in alleviating CAR T cell-mediated CRS and will continue to be used as a component of the first-line treatment.

\section{GM-CSF antagonism}

A strong association between GM-CSF and severity of CRS has been realized in clinical trials [11,12]. This has led to speculation that GM-CSF is a key effector cytokine in facilitating early myeloid activation which results in CRS and neurotoxicity following CART cell therapy. Likewise, a case study conducted by Rosenberg et al. revealed increased serum levels of GM-CSF alongside IL-6 in a colon cancer patient receiving anti-ERBB2 (Her-2/neu) CAR T cells [17]. Based on this rationale, our group addressed this possibility using a humanized mouse model and demonstrated novel neutralizing anti-GM-CSF, respectively, abrogated features of CRS and neuroinflammation [8]. By employing this patient-derived xenograft model that recapitulates aspects of neuropathology reported in clinical 
settings, we further confirmed that GM-CSF blockade yields not only intact functionality but potentially improves cytotoxic efficiency of anti-CD19 CAR T cells, all while reducing BBB disruption.

Even though patient-derived xenograft model supports a role for T cell derived GM-CSF, our study does not negate the participation of CNS resident or other immune-cell types in amplification of GM-CSF or other T cellderived cytokines. Furthermore, we also recognize the effects of GM-CSF in gliosis, and subsequent downstream effects on the neurovascular unit, which have been defined separately in pathogen-associated neuroinflammation model systems [18,19]. Mechanistic studies are currently underway within our research group to define the contribution of specific CNS cell types in mediating neuroinflammation and BBB disruption following CAR T cell therapy. Preservation of antitumor activity paired with ability to restrict BBB-disruption, therefore makes GM-CSF blockade a promising candidate for therapeutic strategy to reduce CRS and neurotoxicity. A Phase II trial with the aim to verify efficacy of combinatory CART19 and lenizulumab approach is also currently in progress.

\section{IL-1 receptor antagonism}

Most updated insights about the role of IL-1 in promoting neurotoxicity in CAR T cell therapy have been derived from murine models $[7,9]$. Production of IL-1 by macrophages has been put forward as a putative mechanism of neurotoxicity in CAR T cells therapy [7,9]. Additionally, using a xenotolerant mouse model, investigators were able to demonstrate Anakinra, a recombinant IL-1 receptor (IL-1R) antagonist, could prevent CAR T cellassociated CRS as well as neurotoxicity [7]. A similar study corroborated IL-1R blockade mitigates severe CRS and neuropathology [9]. Importantly, both studies identified myeloid cells, namely macrophages and monocytes, as the key source of IL-1 cytokine. IL-R1 antagonist (Anakinra) currently US FDA approved for RA was successfully able to attenuate CRS and neurotoxicity syndromes while preserving antitumor efficacy of the CAR T cells in these murine studies. Nevertheless, IL-1 $\beta$ is a fundamental arm of innate immunity and may have immunological consequences in patients with acute-viral infection who are administered immunosuppressants during severe CRS.

\section{Corticosteroids}

The use of corticosteroids following CAR T cell therapy is reserved for clinical cases where toxicities are refractory to IL-6 targeting therapies. Currently, a standard intervention for CRS consists of dexamethasone $10 \mathrm{mg}$ intravenous (IV) every $6 \mathrm{~h}$ or methylprednisolone $1 \mathrm{mg} / \mathrm{kg}$ IV every $12 \mathrm{~h}$ in instances of moderate to high-grade CRS [1,6]. An obvious concern with use of corticosteroids in immunotherapies is its well-known immunosuppressive function. Nevertheless, low dose and short regimen do not impair either cytotoxicity or proliferation of CAR T cells infused in patients. On the other hand, corticosteroids such as dexamethasone and prednisone are reliable for treatment of neurological disease due to their ability to permeate the CNS and elicit robust responsiveness to edema and swelling [6]. Additionally, some investigators have emphasized that antiepileptic drugs are more appropriate for low grade neurotoxicity accompanied by seizures rather than gross use of steroids [1]. Despite existing reservations about broad immunosuppression as well as impairment of $\mathrm{T}$ cell function, corticosteroids remain valuable when paired with first-line of treatments to manage moderate-to-severe CRS.

\section{Small molecule inhibitors}

Due to broad immunosuppressive effects of corticosteroids that can impair antitumor efficacy of $\mathrm{T}$ cell-engaging immunotherapies, small molecule inhibitor such as JAK/STAT inhibitors can offer strategies to disrupt abnormal intracellular signaling that contribute to CRS pathology. With the use of NOD-scid IL2 $\gamma \gamma^{\text {null }}$ (NSG), mouse model bearing primary acute myeloid leukemia that incorporate additional transgenics for IL-3, GM-CSF and stem-cell factor, a recent preclinical study demonstrated Ruxolitinib (a small-molecule inhibitor JAK 1/2) treatment after infusion of CD123-specific CAR T cells prevent development of CRS and long-term survival [20]. IL-6 and GMCSF receptor signaling utilize JAK1 and JAK2, respectively; thus, Ruxolitinib could have relevance in attenuating CRS-driven by these cytokines at an early time point. Likewise, Bruton's tyrosine kinase inhibitor (BTK) inhibitors can directly target leukemic B cells and reduce aberrant cytokine production due to enhanced BTK signaling. Ibrutinib, a BTK inhibitor, currently approved for relapsed chronic lymphocytic leukemia, conferred CRS-free survival and reduced inflammatory cytokines such as IL-6, GM-CSF and IFN- $\gamma$ in a xenograft model of CRS while CD19-specific CAR T cells function remained unaffected [21]. A clinical trial (NCT02640209) that pairs CTL019(anit-CD19 CAR T cells) with ibrutinib in patient with relapsed or refractory chronic lymphocytic leukemia is currently underway and will provide insights on efficacy and durability of BTK inhibitor in managing 
CAR T cell-mediated CRS. However, caution must be still warranted with use of tyrosine kinase inhibitors as some including dasatinib and nilotinib may lead to severe events of pulmonary and cardiovascular damage.

\section{Future perspective: a new era of engineering with interdisciplinary crosstalk}

As immunotherapies become translated into the clinical setting, a balance between efficacy and toxicity management must be achieved. Tremendous efforts have been put forward to use synthetic engineering of $T$ cells, including SUPRA CAR T cells, synNotch receptors and CRIPSPR editing to enhance efficacy and off-the-shelf product feasibility. In contrast, advancement in innovative strategies to ameliorate toxicities associated with immunotherapy has lagged behind. Nonetheless, inhibitory CARs (i-CAR), Tet-On System CARs, Switch CARs, inducible caspase CARs as well as CAR logic gates are emerging strategies with built-in features to reduce toxicities, some of which are being currently tested in trials [2,22]. Similarly, genetic engineering approach like GM-CSF ${ }^{\mathrm{K} / \mathrm{O}}$ CAR $\mathrm{T}$ cells may bear practical function due to enhancement of cytotoxic efficacy combined with reduced GMCSF dependent neurotoxicity. Approaches to circumvent CRS and neurotoxicity syndrome should not just be limited to engineering solutions. Additional insights gained from mechanistic knowledge generate new forms of interventions and engineering feats. Revisiting and drawing knowledge from studies of autoimmune and neurodegenerative diseases can bolster our current understanding of T cell-mediated immunopathology and provide new avenues to improve toxicity management. For example, adhesion molecule-targeting approaches used to overcome excessive cell trafficking into the brain in demyelinating conditions such as multiple sclerosis could be employed in preclinical studies for prevention of neurotoxicity. Furthermore, it is imperative to encourage interdisciplinary efforts across experts from fields such as neuroscience, virology, immunology and oncology. Continuation of these multidisciplinary efforts will help generate effective strategies to avert CRS and neurotoxicity associated with $\mathrm{T}$ cell therapies moving forward.

\section{Financial disclosure \& competing interests}

This work was supported by R01 NS 103212. SS Kenderian is an inventor on patents in the field of CART cell therapy that are licensed to Novartis (under an agreement between Mayo Clinic, University of Pennsylvania, and Novartis). SS Kenderian's laboratory receives research funding from Kite, Gilead, Novartis, Humanigen, Tolero, Lentigen, and Morphosys. The authors have no other relevant affiliations or financial involvement with any organization or entity with a financial interest in or financial conflict with the subject matter or materials discussed in the manuscript apart from those disclosed.

No writing assistance was utilized in the production of this manuscript.

\section{References}

1. Neelapu SS, Tummala S, Kebriaei P et al. Chimeric antigen receptor T cell therapy-assessment and management of toxicities. Nat. Rev. Clin. Oncol. 15(1), 47 (2018).

2. Paucek RD, Baltimore D, Li G. The cellular immunotherapy revolution: arming the immune system for precision therapy. Trends Immunol. 40(4), 292-309 (2019).

3. Yu S, Li A, Liu Q et al. Recent advances of bispecific antibodies in solid tumors. J. Hematol. Oncol. 10(1), 155 (2017).

4. Martinelli G, Boissel N, Chevallier P et al. Complete hematologic and molecular response in adult patients with relapsed/refractory Philadelphia chromosome positive B-precursor acute lymphoblastic leukemia following treatment with blinatumomab: results from a Phase II, single-arm, multicenter study. J. Clin. Oncol. 35, 1795-802 (2017).

5. Kantarjian H, Stein A, Gokbuget $\mathrm{N}$ et al. Blinatumomab versus chemotherapy for advanced acute lymphoblastic leukemia. $N$. Engl. J. Med. 376, 836-847 (2017).

6. Lee DW, Santomasso BD, Locke FL et al. ASTCT consensus grading for cytokine release syndrome and neurological toxicity associated with immune effector cells. Biol. Blood Marrow Transplant. 25(4), 625-638 (2018).

7. Norelli M, Camisa B, Barbiera G et al. Monocyte-derived IL-1 and IL-6 are differentially required for cytokine-release syndrome and neurotoxicity due to CAR T cells. Nat. Med. 24(6), 739 (2018).

8. Sterner RM, Sakemura R, Cox MJ et al. GM-CSF inhibition reduces cytokine release syndrome and neuroinflammation but enhances CAR T cell function in xenografts. Blood 133(7), 697-709 (2019).

9. Giavridis T, van der Stegen SJ, Eyquem J, Hamieh M, Piersigilli A, Sadelain M. CAR T cell-induced cytokine release syndrome is mediated by macrophages and abated by IL-1 blockade. Nat. Med. 24(6), 731 (2018).

10. Schuster SJ, Bishop MR, Tam CS et al. Tisagenlecleucel in adult relapsed or refractory diffuse large B-cell lymphoma. $N$. Engl. J. Med. 380, 45-56 (2019).

11. Locke FL, Ghobadi A, Jacobson CA et al. Long-term safety and efficacy of axicabtagene ciloleucel (anti-CD19 CAR T) in refractory large B-cell lymphoma: a multicenter, single arm, Phase I-II trial. Lancet Oncol. 20, 31-42 (2018). 
12. Abramson JS, Siddiqi T, Palomba ML et al. High durable CR rates and preliminary safety profile for JCAR017 in R/R aggressive b-NHL(TRANSCEND NHL 001 study): a defined composition CD19-directed CART cell product with potential for outpatient administration. J. Clin. Oncol. 36(suppl), 120 (2018).

13. Gust J, Hay KA, Hanafi LA et al. Endothelial activation and blood-brain barrier disruption in neurotoxicity after adoptive immunotherapy with CD19 CAR T cells. Cancer Dis. 7(12), 1404-1419 (2017).

14. Sebastian M, Kiewe P, Schuette W et al. Treatment of malignant pleural effusion with the trifunctional antibody catumaxomab (Removab) (anti-EpCAM $\times$ anti-CD3): results of a Phase I/II study. J. Immunother.32(2), 195-202 (2009).

15. Brudno JN, Maric I, Hartman SD et al. T cells genetically modified to express an anti-B-cell maturation antigen chimeric antigen receptor cause remissions of poor-prognosis relapsed multiple myeloma. J. Clin. Oncol. 36(22), 2267-2280 (2018).

16. Teachey DT, Rheingold SR, Maude SL et al. Cytokine release syndrome after blinatumomab treatment related to abnormal macrophage activation and ameliorated with cytokine-directed therapy. Blood 121(26), 5154-5157 (2013).

17. Morgan RA, Yang JC, Kitano M et al. Case report of a serious adverse event following the administration of $\mathrm{T}$ cells transduced with a chimeric antigen receptor recognizing ERBB2. Mol. Ther. 18(4), 843-851 (2010).

18. Willenbring RC, Jin F, Hinton DJ et al. Modulatory effects of perforin gene dosage on pathogen-associated blood-brain barrier (BBB) disruption. J. Neuroinflam. 13(1), 222 (2016).

19. Huggins MA, Johnson HL, Jin F et al. Perforin expression by CD8 T cells is sufficient to cause fatal brain edema during experimental cerebral malaria. Inf. Immun. 85(5), 00985-00916 (2017).

20. Kenderian SS, Ruella M, Shestova $\mathrm{O}$ et al. Ruxolitinib prevents cytokine release syndrome after CART cell therapy without impairing the anti-tumor effect in a xenograft model. Blood 128(22), Abstract 652 (2016).

21. Ruella M, Kenderian SS, Shestova $\mathrm{O}$ et al. Kinase inhibitor ibrutinib to prevent cytokine-release syndrome after anti-CD19 chimeric antigen receptor T cells for B-cell neoplasms. Leukemia31(1), 246 (2017).

22. Sakemura R, Terakura S, Watanabe $\mathrm{K}$ et al. A Tet-On inducible system for controlling CD19-chimeric antigen receptor expression upon drug administration. Cancer Immunol. Res. 4(8), 658-668 (2016). 
